\title{
Quantitation of green fluorescent protein using a gel-based imaging method.
}

\begin{abstract}
Green fluorescent protein (GFP) is a versatile reporter protein and has been widely used in biological research. However, its quantitation requires expensive equipment such as a spectrofluorometer. In the current study, a gel documentation imaging system using a native polyacrylamide gel for the quantitation of GFP was developed. The assay was evaluated for its precision, linearity, reproducibility, and sensitivity in the presence of Escherichia coli cells and was compared with the spectrofluorometric method. Using this newly established, gelbased imaging technique; the amount of GFP can be quantified accurately.
\end{abstract}

Keyword: Green fluorescent protein; Electrophoresis; Purification. 